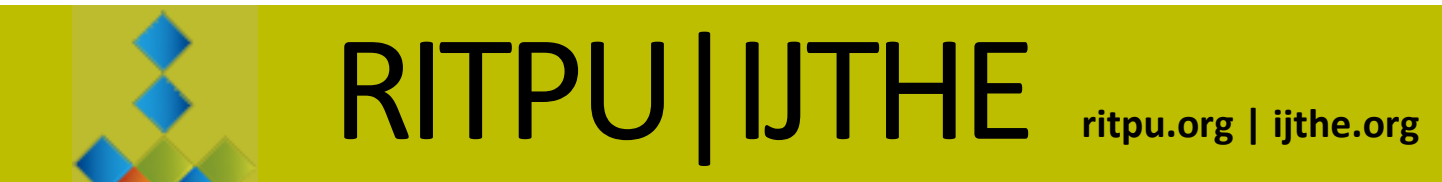

Revue internationale des technologies en pédagogie universitaire International Journal of Technologies in Higher Education

\title{
Inégalités, compétences et conditions numériques
}

Marie-Michèle LEMIEUX Marie-Michele.Lemieux2@uqtr.ca Université du Québec à Trois-Rivières

\section{Inequalities, Skills and Digital Access Conditions}

https://doi.org/10.18162/ritpu-2021-v18n1-14

\section{Mis en ligne : 16 février 2021}

\section{Résumé}

Dans cet article, nous proposons une réflexion sur des enjeux liés au contexte d'enseignementapprentissage mobilisant diverses modalités de formation à distance imposé aux enseignants et aux étudiants pendant la pandémie de COVID-19. Nous nous intéressons particulièrement au développement des compétences numériques des étudiants universitaires et aux risques de voir émerger des inégalités numériques. En nous appuyant sur des résultats d'une recherche récente, nous déterminons des difficultés susceptibles d'être vécues par les étudiants dans leur apprentissage en contexte numérique. Ces difficultés nous amènent à proposer des conditions à mettre en place pour favoriser l'apprentissage numérique des étudiants.

\section{Mots-clés}

Technologies numériques, compétences numériques, inégalités numériques, pédagogie universitaire, modalités de formation à distance

\section{Abstract}

In this paper, we present issues related to distance teaching-learning context imposed to teachers and students during the COVID-19 pandemic. We are interested in development of university students' digital skills and the risks of digital inequalities emergence. Based on recent research findings, we identify some difficulties experienced by students with their learning in a digital context. These difficulties lead us to propose conditions to enhance digital learning.

\section{Keywords}

Digital technologies, digital skills, digital inequalities, university teaching and learning, distance learning methods 


\section{Inégalités, compétences et conditions numériques}

La pandémie de COVID-19 a brusquement amené la plupart des enseignants à revoir leurs pratiques pédagogiques afin d'effectuer un transfert de l'enseignement en présence à l'enseignement à distance. En effet, alors que les milieux d'enseignement ont dû fermer leurs portes pour respecter les règles sociosanitaires, l'introduction de technologies numériques s'est imposée pour permettre la continuité des activités d'enseignement-apprentissage. Dans le contexte universitaire qui nous intéresse, cette situation met en exergue certains enjeux liés à l'intégration des technologies numériques en contexte pédagogique. On compte parmi ces enjeux la disparité dans le taux d'adoption des technologies numériques chez les enseignants universitaires (Collin, 2016) ainsi que le temps (Hobbs et al., 2017; Margaryan et al., 2011) et le soutien (Lameul, 2012) nécessaires pour les adopter. Alors que c'est dans l'urgence que les enseignants ont dû recourir aux technologies numériques pour assurer leur fin de trimestre de l'hiver 2020, il est loisible de penser que ces enjeux demeurent encore présents pour le trimestre actuel d'automne et ceux à venir, pour lesquels un déroulement à distance a d'ores et déjà été annoncé. Conséquemment, certains risques découlent de cette situation. Un des risques encourus réside dans la reproduction de contextes d'apprentissage conduisant vers des inégalités numériques chez les étudiants qui possèdent de manière inégale des compétences dites numériques. C'est dans l'optique de réduire les possibles inégalités numériques que notre réflexion met en lumière l'importance de soutenir le développement des compétences numériques chez les étudiants. Cette réflexion nous semble nécessaire dans le contexte actuel, considérant le déploiement à grande échelle et pour une durée indéterminée de pratiques pédagogiques «à distance » pour assurer la continuité pédagogique. Elle prend assise sur de récents travaux par lesquels nous nous sommes intéressés aux pratiques pédagogiques intégrant des technologies numériques et à leur apport au développement de compétences numériques d'étudiants universitaires (Lemieux, 2020).

Dans un premier temps, nous situons la problématique sous-tendant notre réflexion au regard des contextes d'apprentissage susceptibles de reproduire des inégalités numériques et du développement des compétences numériques des étudiants universitaires. Dans un deuxième temps, nous présentons brièvement une étude de cas qui visait à explorer l'apport de pratiques pédagogiques intégrant des technologies numériques au développement des compétences numériques des étudiants. Dans un troisième temps, à partir des résultats dégagés de cette étude, nous associons au contexte actuel des difficultés rencontrées par les étudiants dans les situations d'enseignement-apprentissage soutenues par des technologies numériques. Également, afin de fournir des pistes pour contrer ces difficultés, nous proposons des conditions pour favoriser l'apprentissage en contexte numérique et soutenir le développement des compétences numériques des étudiants ainsi que, ultimement, leur insertion sociale et professionnelle. Ainsi, cette réflexion trace le parallèle entre la situation pandémique actuellement vécue et les résultats de notre recherche qualitative.

\section{La possible émergence d'inégalités numériques chez les étudiants universitaires}

Dans le contexte d'urgence, plusieurs enseignants et étudiants universitaires ont rapidement eu à adopter et à s'approprier un nouvel environnement d'enseignement-apprentissage " à distance ». De manière réactive, maintes ressources ont été mises en place par les universités et organismes pour accompagner les enseignants dans cette transition vers les modalités de formation à distance et dans le développement de leurs compétences technologiques. En effet, les enseignants qui n'avaient pas d'historique et d'expérience en enseignement en ligne ont été contraints de 
développer de nouvelles façons d'enseigner, notamment par l'utilisation d'une plateforme de visioconférence, l'exploitation accrue de l'environnement numérique d'apprentissage institutionnel ou l'expérimentation de nouvelles technologies numériques. Ainsi, pour favoriser cette rapide et nécessaire appropriation, les équipes pédagogiques ont développé des ressources destinées à soutenir les enseignants universitaires afin qu'ils puissent poursuivre leur enseignement et offrir un encadrement adéquat à leurs étudiants. De l'accompagnement individualisé, des formations multiples, des séances de questions-réponses, des guides et procéduriers, etc. ont été proposés aux enseignants. Par ailleurs, on aura rapidement vu apparaître les appellations "prestation virtuelle » et " enseignement en non-présentiel » qui se sont substituées à celle de "formation à distance » qui implique diverses caractéristiques difficiles à appliquer dans l'état actuel. Conséquemment, dans ce contexte, nous soulignons le risque exacerbé pour les étudiants d'être confrontés à de nouvelles difficultés, liées notamment au fait qu'ils ne sont pas tous familiarisés sinon habiles avec les technologies numériques proposées pour soutenir leur apprentissage et que les conditions d'une formation à distance de qualité n'ont pas toujours pu être réunies. Par ailleurs, soulignons que le mythe lié aux « apprenants numériques » persiste encore, bien que la littérature scientifique suggère d'aller audelà du débat sur les prédispositions générationnelles (Roy et al., 2018). C'est au regard de la présence de conditions et de considérations à ces égards que notre appréhension s'amorce. Précisément, nous nous questionnons sur le soutien offert aux étudiants pour favoriser leur apprentissage dans cette variabilité de modalités de formation à distance. Les résultats de notre recherche nous amènent à proposer que les difficultés vécues par les étudiants dans un contexte d'apprentissage numérique donné sont susceptibles de se reproduire dans le contexte de formation à distance et davantage dans la situation que nous qualifions encore d'urgence. Ainsi, les préoccupations à l'égard des étudiants et de leur capacité à s'adapter à l'apprentissage numérique nous apparaissent prioritaires pour déterminer des conditions à mettre en place afin de limiter les risques inhérents à la situation actuelle. En nous référant aux travaux de Collin (2013), nous établissons la reproduction de contextes conduisant vers des inégalités numériques comme un risque encouru. Ces inégalités peuvent être associées aux trois niveaux suivants :

- L'avoir (l'accès aux technologies numériques);

- Le savoir (les compétences et les usages technologiques possibles);

- Le pouvoir, c'est-à-dire « la capacité, pour un individu, de mettre à profit les usages et les compétences technologiques pour servir ses intérêts et son capital individuel ». (p. 3)

En ce qui concerne l'avoir, il est loisible de se rappeler que tous les étudiants ne possèdent pas nécessairement les appareils électroniques nécessaires pour suivre de manière adéquate l'ensemble de leurs cours universitaires en ligne (visioconférence, consultation des environnements numériques d'apprentissage, lecture de documents numériques, etc.). Aussi, notons que certains services de prêts d'équipements informatiques par les universités ont été suspendus pendant la pandémie. Alors qu'au Québec des mesures gouvernementales ont permis d'assurer l'accès aux technologies numériques pour les élèves des niveaux scolaires primaire et secondaire, à notre connaissance, de telles dispositions sont encore peu présentes dans les établissements postsecondaires. Soulignons également qu'advenant que des étudiants puissent avoir une aisance financière suffisante pour s'offrir une connexion Internet, l'accès de qualité - à haute vitesse - n'est pas assuré dans toutes les régions, ce qui vient limiter les usages possibles des technologies numériques (Adams Becker et al., 2017; Brotcorne, 2019). Ainsi, avant même de s'intéresser aux compétences numériques, il est nécessaire de lever cette première barrière liée à l'avoir. 
Au deuxième niveau, comme pour l'enseignant, l'étudiant en contexte numérique doit mobiliser des compétences de base pour utiliser des appareils informatiques et des logiciels. Or, dans le contexte actuel, certains étudiants utiliseront pour la première fois des technologies numériques en contexte d'apprentissage. À cet effet, il est erroné de penser que le seul fait d'exposer les étudiants aux technologies numériques leur permettra de développer les compétences nécessaires pour les utiliser (Murray et Pérez, 2014). À qui reviendra la tâche de former les étudiants à l'utilisation adéquate des technologies mises à leur disposition? Les étudiants savent-ils vers quelles ressources ou quels services se tourner pour être soutenus dans le développement de leurs compétences technologiques? Bien que nécessaires pour lever le deuxième niveau de barrières et limiter les inégalités de l'ordre du savoir, les compétences technologiques ne sauraient toutefois être suffisantes pour soutenir l'apprentissage (Littlejohn et al., 2012; Secker, 2011) et favoriser l'appropriation des technologies numériques mises à la disposition des étudiants (Massin, 2019). D'ailleurs, même si des conditions limitant les situations susceptibles de reproduire des inégalités numériques des deux premiers niveaux sont mises en place, elles se cantonnent dans la perspective «enseigner par le numérique ». Cette perspective évacue les considérations liées au pouvoir, que nous associons, au-delà de l'axe technologique, aux diverses sphères de compétences mobilisées en contexte numérique (voir l'annexe).

Par ailleurs, si certains étudiants possèdent les compétences technologiques requises pour utiliser adéquatement les appareils numériques, tous ne savent pas apprendre en contexte numérique, c'est-à-dire, par exemple, collaborer et communiquer avec le numérique, gérer leur environnement numérique et assurer l'équilibre nécessaire entre les études et les loisirs. Ils ne l'ont pas appris (Roy et al., 2018). Or, nous associons ces compétences numériques à la capacité des étudiants d'interagir et de s'intégrer socialement et professionnellement en contexte numérique (le niveau du pouvoir). De plus, contrairement à l'étiquette de «natifs du numérique » accolée aux étudiants par Prensky (2001), tous les étudiants universitaires de la génération actuelle ne sont pas nécessairement habiles à utiliser de manière optimale les technologies numériques au service de leur apprentissage (Littlejohn et al., 2012; Secker, 2011). Plus encore, dans le contexte actuel, les étudiants n'ont pas fait le choix de suivre des cours à distance. Ainsi, selon leurs profils variés en matière d'acquis et de compétences numériques, ils sont susceptibles d'éprouver des difficultés d'apprentissage liées au contexte numérique.

Au regard des niveaux d'inégalités numériques encourues, si les inégalités de l'ordre de l'avoir peuvent être enrayées, en assurant l'accès aux technologies numériques, et celles de l'ordre du savoir limitées par le soutien au développement des compétences technologiques des étudiants, notre réflexion se positionne particulièrement sur le plan des inégalités de l'ordre du pouvoir. Plus précisément, nous désirons mettre en évidence l'importance à accorder à l' " enseigner $a u$ numérique ». Selon cette perspective, une implication des établissements et des enseignants s'avère essentielle pour créer des conditions favorables à la réussite des étudiants dans leur apprentissage numérique (Margaryan et al., 2011). Dans cet article, nous nous attardons principalement à l'implication enseignante. Cette implication réside dans ce qu'Araújo-Oliveira (2012) désigne comme la « fonction sociale [de l'enseignant] qui consiste à aider l'individu à percevoir et à interpréter son environnement » (p. 72), ce qui est selon Lenoir (2009) un élément essentiel du processus d'enseignement-apprentissage.

Nous proposons une réflexion pédagogique sur les compétences numériques susceptibles d'être sollicitées chez les étudiants dans les activités d'enseignement-apprentissage en contexte numérique et sur les conditions permettant d'en favoriser le développement. La mise en place de certaines conditions est susceptible de limiter les difficultés que peuvent rencontrer les étudiants 
dans le contexte à distance actuellement vécu et d'ainsi lever les barrières se dressant dans l'apprentissage numérique. Cette réflexion repose sur ce que nous avons réalisé dans une étude de cas en nous intéressant à l'apport de technologies numériques au développement des compétences numériques d'étudiants universitaires. Dans les prochaines sections, nous présentons brièvement la recherche ainsi que les résultats inhérents aux difficultés rencontrées par des étudiants universitaires. Ces résultats viennent appuyer nos propositions de conditions à mettre en place en formation à distance, de manière à limiter les contextes reproduisant les inégalités numériques tout en favorisant le développement de compétences numériques.

\section{Anticiper et évaluer les pratiques pédagogiques pour limiter les inégalités numériques}

Les résultats d'une étude de cas unique réalisée dans un cours universitaire ont permis de déterminer, au regard des perceptions des étudiants et de leur enseignant ainsi que des observations de la chercheuse, des difficultés perçues en contexte d'apprentissage numérique ainsi que des conditions pour les contrer (Lemieux, 2020). Bâtie selon une adaptation du modèle de l'intervention éducative proposé par Lenoir et ses collègues (Lenoir, 2009, 2014; Lenoir et al., 2001), cette recherche nous a amenée à étudier les activités d'enseignement-apprentissage intégrant des technologies numériques, le manuel numérique précisément. Nous nous sommes intéressée aux pratiques pédagogiques anticipées, effectives et réflexives d'un cours donné, s'échelonnant sur un trimestre universitaire. Pour collecter les perceptions des acteurs (l'enseignant universitaire et ses étudiants) selon les diverses pratiques, des méthodes de collecte de données ont été associées aux trois phases de l'intervention éducative - préactive, interactive et postactive. La figure 1 permet d'illustrer comment la collecte de données a permis de couvrir les trois phases de l'intervention éducative en sollicitant les acteurs au regard des pratiques pédagogiques anticipées, effectives et réflexives inhérentes à l'utilisation de technologies numériques.

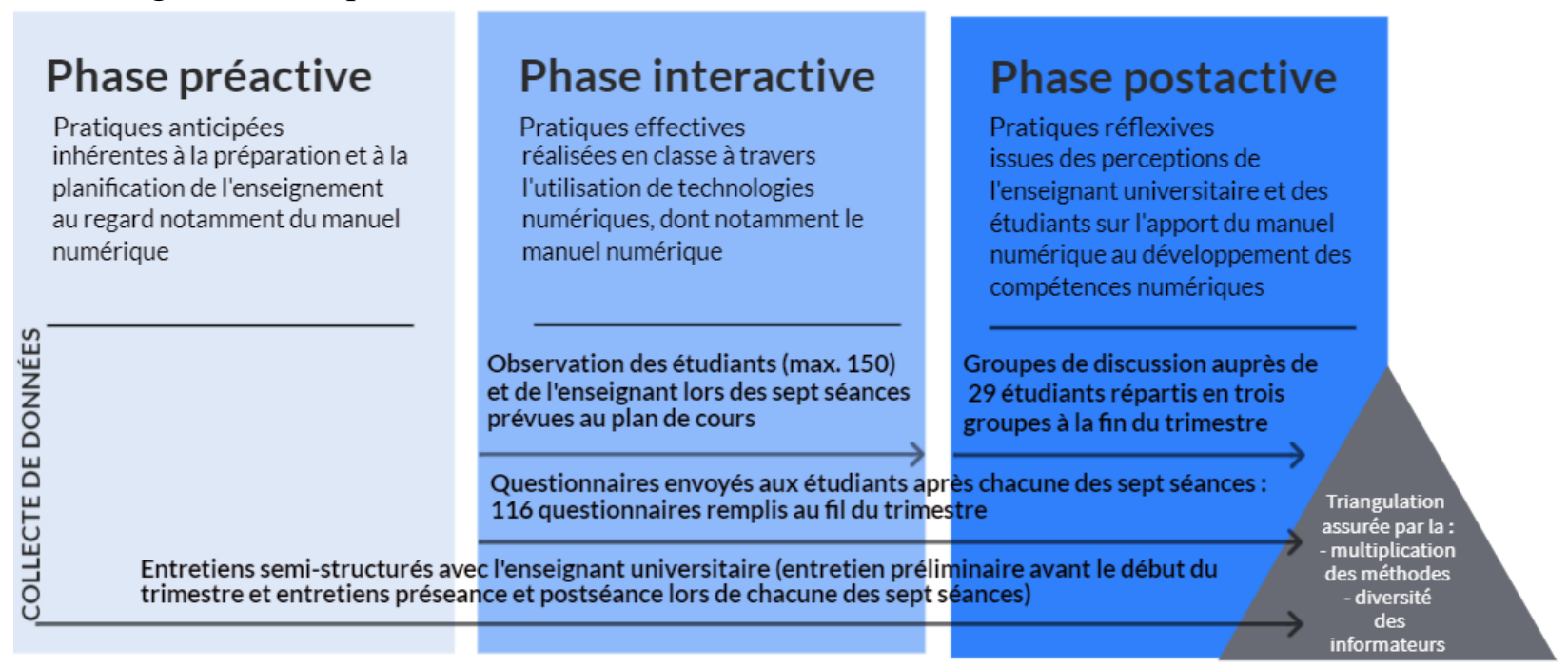

\section{Figure 1}

Les méthodes de collecte de données associées aux trois phases de l'intervention éducatives afin de solliciter les acteurs au regard des pratiques anticipées, effectives et réflexives

Ainsi, appliquée tout au long des sept séances en classe prévues au plan de cours, la méthodologie de recherche qualitative a permis de recueillir un grand nombre de données issues : 
- d'entretiens semi-structurés avec l'enseignant universitaire (phases préactive et postactive). L'entretien préliminaire, avant le début du trimestre, ainsi que les sept entretiens préséances visaient les pratiques anticipées inhérentes à la préparation et à la planification de l'enseignement ainsi qu'aux conditions mises en place au regard de l'utilisation des technologies numériques. Par les sept entretiens postséances, l'enseignant était amené à porter un regard réflexif sur les pratiques effectives et leur apport au développement des compétences numériques des étudiants ainsi que sur les conditions qui pourraient être mises en place pour le favoriser;

- de groupes de discussion avec 29 étudiants universitaires (phase postactive). Dans les groupes de discussion, les étudiants étaient invités à partager leurs perceptions sur l'apport des technologies numériques utilisées dans le développement de leurs compétences numériques ainsi que sur les difficultés rencontrées avec celles-ci dans leur apprentissage;

- de questionnaires en ligne destinés aux étudiants (phases interactive et postactive). Envoyé par courriel à tous les étudiants inscrits au terme de chacune des sept séances, le questionnaire a recueilli des données descriptives secondaires permettant de confronter les données issues de l'observation et des propos partagés dans les entretiens afin d'assurer la description des pratiques effectives. Les étudiants étaient notamment invités à indiquer les fonctionnalités numériques utilisées à partir d'une grille d'items à cocher. Une question ouverte permettait d'établir des difficultés ou limites liées à l'utilisation des technologies;

- d'observations réalisées par la chercheuse en salle de classe (phase interactive). L'observation en situation a été effectuée principalement autour des activités d'enseignement-apprentissage impliquant l'utilisation des technologies numériques. Tous les étudiants présents aux séances en salle de classe ainsi que l'enseignant étaient susceptibles d'être observés dans le cadre de cette recherche.

Par la multiplication de ces méthodes, utilisées auprès d'une diversité d'informateurs - les acteurs -, nous avons assuré la triangulation de nos données permettant d'étudier l'apport perçu des pratiques pédagogiques intégrant des technologies numériques au développement des compétences numériques. Comme cette recherche visait la compréhension approfondie d'un phénomène, le traitement et l'analyse des données ont été de type qualitatif. Nous avons effectué une analyse dite délibératoire (Savoie-Zajc, 2018), c'est-à-dire que nous avons extrait nos données pour ensuite les regrouper à partir de catégories issues de notre cadre conceptuel ou émergeant des données collectées. Ces données catégorisées ont ensuite fait l'objet d'une recontextualisation, pour reprendre les propos de Savoie-Zajc (2018), de manière à faire ressortir les sens qui s'en dégagent. En nous intéressant à la fois aux pratiques anticipées, effectives et réflexives, nous avons pu dégager des points de rupture, ou ce que nous avons appelé des « prises de conscience pédagogiques » dans les propos de l'enseignant, ainsi que des éléments de convergence et de divergence entre les perceptions des acteurs impliqués. Parmi les résultats obtenus, notre analyse met en lumière une mobilisation perçue à la fois par les étudiants et par l'enseignant de certaines compétences numériques. Ainsi, les compétences mobilisées s'inscrivent notamment dans l'utilisation des technologies numériques; elles sont aussi associées à l'apprentissage numérique et au bien-être numérique. Selon le référentiel retenu pour notre étude (Beetham, 2016), les compétences liées à l'apprentissage numérique se réfèrent à la capacité d'exploiter les possibilités d'apprentissage numérique et d'en bénéficier, alors que le bien-être vise la capacité de veiller à la santé, à la sécurité, aux relations et à l'équilibre travailvie personnelle dans les environnements numériques. Nous associons particulièrement ces deux sphères de compétences numériques aux pratiques liées à l'« enseigner au numérique ». 
Dans l'étude, des conditions ont été dégagées afin de lever les difficultés rencontrées par les étudiants dans les activités pédagogiques intégrant des technologies numériques. Ces conditions visent, d'une part, à favoriser l'apprentissage et, d'autre part, à limiter la reproduction de contextes conduisant vers des inégalités numériques. Les conditions émergent du regard réflexif des acteurs impliqués dans les situations d'enseignement-apprentissage. La prochaine section expose les types de difficultés que nous avons pu observer ou relever dans les propos des acteurs et que nous transposons à la situation actuelle. Nous suggérons ensuite des conditions pour limiter ces difficultés et favoriser l'apprentissage en formation « à distance ».

\section{Des difficultés et des conditions pour favoriser l'apprentissage numérique}

Au regard des résultats de notre étude et inspirée par les travaux d'Araújo-Oliveira (2012), nous pouvons associer des difficultés susceptibles d'être vécues par les étudiants dans leur apprentissage numérique à trois types: 1) les difficultés pédagogiques; 2) les difficultés didactiques; 3) les difficultés organisationnelles. Le tableau 1 présente ces trois types de difficultés et leur définition. Aussi, pour chacun des types de difficultés, nous proposons quelques exemples inspirés de nos résultats, pour lesquels nous associons le niveau de risque encouru en matière d'inégalités numériques - nous reprenons ici la classification proposée par Collin (2013).

\section{Tableau 1}

Les trois types de difficultés susceptibles d'être vécues chez les étudiants dans leur apprentissage numérique : exemples et niveaux d'inégalités numériques

\begin{tabular}{|c|c|c|c|}
\hline $\begin{array}{l}\text { Types } \\
\text { de difficultés }\end{array}$ & Définition & Exemples de difficultés & $\begin{array}{l}\text { Niveaux } \\
\text { de risque }\end{array}$ \\
\hline \multirow{5}{*}{ Pédagogiques } & \multirow{5}{*}{$\begin{array}{l}\text { Les stratégies } \\
\text { d'enseignement ou } \\
\text { d'apprentissage déployées } \\
\text { pour favoriser l'utilisation } \\
\text { des technologies } \\
\text { numériques }\end{array}$} & $\begin{array}{l}\text { Méconnaissance des technologies numériques } \\
\text { utilisées et de leurs fonctionnalités }\end{array}$ & savoir \\
\hline & & $\begin{array}{l}\text { Manque d'habiletés avec les technologies } \\
\text { numériques utilisées }\end{array}$ & savoir \\
\hline & & $\begin{array}{l}\text { Manque d'adaptation des stratégies d'apprentissage } \\
\text { aux technologies numériques utilisées }\end{array}$ & pouvoir \\
\hline & & $\begin{array}{l}\text { Inconfort à communiquer par les outils de } \\
\text { communications collaboratifs (ex. forum) }\end{array}$ & pouvoir \\
\hline & & $\begin{array}{l}\text { Gestion complexe du temps et des ressources selon } \\
\text { les modalités de formation à distance utilisées - } \\
\text { équilibre étude-vie personnelle }\end{array}$ & pouvoir \\
\hline \multirow{2}{*}{ Didactiques } & \multirow{2}{*}{$\begin{array}{l}\text { Les ressources mises à la } \\
\text { disposition des étudiants } \\
\text { pour soutenir leur } \\
\text { apprentissage }\end{array}$} & $\begin{array}{l}\text { Contraintes liées à la navigation dans } \\
\text { l'environnement numérique d'apprentissage }\end{array}$ & avoir \\
\hline & & $\begin{array}{l}\text { Complexité liée à la multiplicité des technologies } \\
\text { numériques utilisées }\end{array}$ & pouvoir \\
\hline \multirow{3}{*}{ Organisationnelles } & \multirow{3}{*}{$\begin{array}{l}\text { L'infrastructure entourant } \\
\text { l'utilisation des } \\
\text { technologies numériques }\end{array}$} & Nécessité d'avoir un appareil informatique & avoir \\
\hline & & $\begin{array}{l}\text { Limites imposées par l'appareil utilisé par } \\
\text { les étudiants }\end{array}$ & avoir \\
\hline & & Mauvaise qualité de la connexion Internet & avoir \\
\hline
\end{tabular}

Au-delà des difficultés liées intrinsèquement à l'accès (avoir) et aux compétences technologiques (savoir), il ressort dans les difficultés établies au tableau 1 des éléments liés aux stratégies d'apprentissage numérique et au bien-être numérique. Ces difficultés risquent de dresser des barrières de l'ordre du pouvoir. Dans notre compréhension des compétences numériques, ce 
« pouvoir » représente un levier favorisant l'intégration sociale et professionnelle ainsi que la réussite éducative (HabiloMédias, s.d.; Koltay, 2011; Roy et al., 2018). C'est donc pour limiter les inégalités numériques possibles, de tout ordre, que nous suggérons des conditions à mettre en place dans les pratiques pédagogiques en contexte de formation à distance. Inspirée par les travaux de Depover et Strebelle (1997), nous avons classifié les conditions selon trois catégories: technique, méthodologique et relationnelle. Elles sont présentées au tableau 2 et illustrées à l'aide d'exemples, qui ne sauraient être exhaustifs.

\section{Tableau 2}

Les trois catégories de conditions à mettre en place dans les pratiques pédagogiques en contexte de formation à distance soutenues par des exemples

\begin{tabular}{ll} 
Catégories & Explication \\
de conditions & Exemples \\
\hline
\end{tabular}

Soutien possible pour favoriser l'utilisation des technologies

Technique numériques proposées dans les modalités de formation à distance et infrastructure en place

Proposer un moment pour vérifier les connexions, logiciels et autres technologies numériques

Fournir des procéduriers écrits ou des vidéos sur l'utilisation des principales technologies numériques

Proposer une formation/mise à niveau sur les compétences technologiques

Déterminer les ressources offertes dans l'établissement d'enseignement pour accéder au soutien technologique

Assurer l'alignement technopédagogique des activités et des évaluations

Expliquer le choix des technologies numériques retenues

Soutien visant l'appropriation

Méthodologique des technologies numériques et leur utilisation par les étudiants

Proposer une structure cohérente et facile à suivre dans les activités présentées dans l'environnement numérique pour guider l'étudiant dans son cheminement et sa progression dans les tâches demandées

Modéliser l'utilisation des technologies numériques

Créer un premier contact en mode synchrone pour déterminer les attentes de l'enseignant et des étudiants

Prendre le temps de vérifier l'installation et l'utilisation des requis technologiques pour rassurer les étudiants

Mettre en place des activités sollicitant des interactions entre l'étudiant et l'enseignant ainsi qu'entre les étudiants

Soutien possible pour favoriser l'ouverture au changement face Relationnelle aux modalités de formation à distance ainsi que la motivation et l'engagement des étudiants
Proposer régulièrement des activités sur l'environnement numérique d'apprentissage pour inciter les étudiants à le fréquenter

Proposer des solutions alternatives en mode asynchrone pour limiter les barrières liées à l'accès (p. ex., fournir les enregistrements des séances synchrones)

Mettre en place des moyens de communication non officiels (p. ex., une page Facebook pour partager des informations relatives à la vie professionnelle future des étudiants)

Informer les étudiants des ressources et des divers services offerts en ligne par l'université pour les soutenir 
Au regard des trois catégories de conditions et des exemples proposés, nous mettons en évidence le nécessaire partage de responsabilités entre les services offerts par l'établissement et le rôle de l'enseignant. En effet, alors que les conditions techniques peuvent être assurées en partie par l'établissement, celles qui sont issues des catégories méthodologique et relationnelle relèvent davantage de l'enseignant. Afin de favoriser la détermination des conditions pertinentes et leur mise en œuvre dans le contexte d'enseignement-apprentissage actuel, nous suggérons la tenue d'une réflexion pédagogique. Comme nous l'avons mis en application dans l'étude présentée dans cet article, cette réflexion se veut non seulement préalable - ou préactive pour reprendre les termes de l'intervention éducative - à toute situation d'enseignement-apprentissage mobilisant des modalités de formation à distance, mais également réflexive - postactive - afin de porter un regard sur les pratiques effectives et les difficultés en émergeant. Cette façon de procéder nous apparaît encore plus nécessaire dans le contexte actuel où les exigences liées à la formation à distance ne sont pas toujours réunies. Ainsi, en étant conscients des différentes difficultés possiblement vécues par les étudiants et par la mise en place de conditions, l'enseignant et l'établissement contribueront certainement à diminuer les contextes reproduisant des inégalités numériques, qu'elles soient de l'ordre de l'avoir, du savoir ou du pouvoir (Collin, 2013) et favoriseront le développement des compétences numériques.

Les conditions techniques permettent notamment de lever les barrières d'accès tout en favorisant le développement de compétences technologiques des étudiants. Les conditions méthodologiques contribuent également à favoriser le développement des compétences technologiques, davantage en matière de productivité, ainsi qu'une appropriation du numérique pour soutenir l'apprentissage. Ainsi, l'explication des choix des technologies numériques, la structure des activités et des tâches proposées aux étudiants et le respect de l'alignement technopédagogique (Meyer et al., 2019) sont des exemples de conditions qui visent à mieux étayer les potentialités ou possibilités d'apprentissage - liées aux technologies utilisées. Le partage des stratégies d'apprentissage par l'enseignant ou encore de celles que déploient les étudiants peut s'avérer riche et bénéfique pour tous. Ce partage vient du même coup limiter les frustrations qui peuvent découler de l'utilisation des technologies numériques (Pierard et al., 2019). Enfin, les conditions relationnelles nous apparaissent essentielles pour assurer une présence à distance, comme le définit Jézégou (2010), en permettant des interactions, dès le premier contact, entre les étudiants et avec l'enseignant. Plus encore, en établissant des attentes claires en ce qui concerne le déroulement du cours selon les modalités retenues, l'enseignant favorise la perception de l'étudiant envers les compétences à atteindre (Chekour et al., 2015). Également, cela permet à l'enseignant de comprendre comment les étudiants désirent utiliser les technologies mises à leur disposition (Pierard et al., 2019). Ces conditions ont toute leur importance dans le contexte de la pandémie, alors que les étudiants sont susceptibles de vivre davantage de stress, notamment par le fait qu'en étant hors campus, ils n'ont plus les mêmes repères en ce qui concerne leur vie universitaire, les services accessibles, etc. Pour faciliter ce repérage, l'enseignant peut mettre à leur disposition des ressources offertes en ligne pour rejoindre leurs besoins, par exemple liés aux ressources de la bibliothèque ou encore à la gestion de leur anxiété occasionnée par les nouvelles modalités d'apprentissage. Ainsi, par ces conditions, l'enseignant contribue à minimiser les inégalités numériques chez les étudiants et à soutenir de manière plus globale et transversale le développement de leurs compétences dans l'optique de favoriser l'insertion sociale et professionnelle en contexte numérique. 


\section{Conclusion}

Par cette réflexion, nous avions le désir de souligner une problématique liée aux risques d'émergence d'inégalités numériques dans le contexte actuel de formation mobilisant des modalités «à distance» pour assurer la continuité pédagogique dans le respect des règles sociosanitaires relatives à la pandémie de COVID-19. En nous appuyant sur les résultats d'une recherche récente, nous avons associé au contexte actuel des difficultés perçues dans l'apprentissage numérique des étudiants ainsi que des conditions à mettre en place pour les contrer. Également, alors que l'importance accordée au développement des compétences numériques nous semble encore sous-estimée dans les milieux universitaires, nous avons souligné le besoin de les reconnaître dans leur globalité afin de notamment réduire les inégalités numériques de l'ordre de l'avoir, du savoir et du pouvoir. Ces inégalités numériques guettent les étudiants dans ce contexte d'apprentissage essentiellement à distance qui est appelé à rester pour une durée indéterminée et qui ne représente pas nécessairement leur idéal de formation universitaire.

Sur une note plus positive, considérant que dans les dernières années la présence des technologies numériques demeurait en marge du discours pédagogique (Albero, 2011; Lameul, 2012; Newman et Beetham, 2017), il est loisible de penser que déjà quelques mois après le début de cette pandémie, le contexte actuel aura permis de tisser des rapprochements entre la pédagogie et l'utilisation du numérique en mettant en lumière divers enjeux et préoccupations pour mettre la technologie au service de l'enseignement et de l'apprentissage. Plus près de nous, au Québec, il est souhaitable que cette situation propulse l'orientation première du Plan d'action numérique en éducation et en enseignement supérieur qui est de soutenir le développement des compétences numériques des jeunes et des adultes (Ministère de l'Éducation et de l'Enseignement supérieur, 2018, p.25), encadrée par le récent Cadre de référence de la compétence numérique (Ministère de l'Éducation et de l'Enseignement supérieur, 2019).

\section{Références}

Adams Becker, S., Cummins, M., Davis, A., Freeman, A., Hall Giesinger, C. et Ananthanarayanan, V. (2017). NMC Horizon report: 2017 higher education edition. The New Media Consortium. http://library.educause.edu/...

Albero, B. (2011). Le couplage entre pédagogie et technologies à l'université : cultures d'action et paradigmes de recherche. Revue internationale des technologies en pédagogie universitaire, 8(1-2), 11-21. https://doi.org/10.18162/ritpu.2011.190

Araújo-Oliveira, A. (2012). Étude des pratiques d'enseignement en sciences humaines au primaire : le cas des futurs enseignants en contexte de formation en milieu de pratique au Québec. Nouveaux cahiers de la recherche en éducation, 15(2), 64-96. https://doi.org/10.7202/1018457ar

Beetham, H. (2019). Digital capabilities: The six elements defined. Jisc. http://repository.jisc.ac.uk/...

Brotcorne, P. (2019). Pour une approche systémique des inégalités numériques parmi les jeunes en âge scolaire. Nouveaux cahiers de la recherche en éducation, 21(3), 135-154. https://doi.org/10.7202/1067712ar 
Chekour, M., Chaali, R., Laafou, M. et Janati-Idrissi, R. (2015). Impact des théories de la motivation sur l'apprentissage dans le contexte scolaire. EpiNet, (174). http://epi.asso.fr/...

Collin, S. (2013). Les inégalités numériques en éducation : une synthèse. Adjectif.net, 2013(T4). http://adjectif.net/...

Collin, S. (2016, avril). L'adoption du numérique par les enseignants universitaires : où en est-on? Quelques éléments de réflexion [diaporama]. Webinaire du GRIIP, Québec, Canada. http://pedagogie.uquebec.ca/...

Depover, C. et Strebelle, A. (1997). Un modèle et une stratégie d'intervention en matière d'intégration des TIC dans le processus éducatif. Dans L. O. Pochon et A. Blanchet (dir.), L'ordinateur à l'école : de l'introduction à l'intégration (p. 73-98). Institut de recherche et de documentation pédagogique.

HabiloMédias. (s.d.). Les fondements de la littératie numérique. Récupéré le 29 janvier 2021 de http://habilomedias.ca/...

Hobbs, R., Ranieri, M., Markus, S., Fortuna, C., Zamora, M. et Coiro, J. (2017). Digital literacy in higher education: A Report [document de travail]. Media Education Lab. http://mediaeducationlab.com/...

Jézégou, A. (2010). Créer de la présence à distance en e-learning. Cadre théorique, définition et dimensions clés. Distances et savoirs, 8(2), 257-274. http://cairn.info/revue-distances-et-savoirs...

Koltay, T. (2011). The media and the literacies : media literacy, information literacy, digital literacy. Media, Culture and Society, 33(2), 211-221. https://doi.org/10.1177/0163443710393382

Lameul, G. (2012). Travailler sa posture professionnelle pour mieux aborder les situations pédagogiques complexes. Distances et médiations des savoirs, (11). https://doi.org/10.4000/dms.1127

Lemieux, M.-M. (2020). Apport du manuel numérique intégré à une intervention éducative sur le développement de compétences numériques d'étudiants universitaires [thèse de doctorat inédite]. Université du Québec à Trois-Rivières, Canada.

Lenoir, Y. (2009). L'intervention éducative, un construit théorique pour analyser les pratiques d'enseignement. Nouveaux cahiers de la recherche en éducation, 12(1), 9-29. https://doi.org/10.7202/1017474ar

Lenoir, Y. (2014). Les médiations au cœur des pratiques d'enseignement-apprentissage : une approche dialectique. Groupéditions.

Lenoir, Y., Rey, B., Roy, G.-R. et Lebrun, J. (2001). Le manuel scolaire et l'intervention éducative : regards critiques sur ses apports et ses limites. Éditions du CRP.

Littlejohn, A., Beetham, H. et McGill, L. (2012). Learning at the digital frontier: A review of digital literacies in theory and practice. Journal of Computer Assisted Learning, 28(6), 547-556. https://doi.org/10.1111/j.1365-2729.2011.00474.x 
Margaryan, A., Littlejohn, A. et Vojt, G. (2011). Are digital natives a myth or reality? University students' use of digital technologies. Computers \& Education, 56(2), 429-440. https://doi.org/10.1016/j.compedu.2010.09.004

Massin, S. (2019). L'utilisation de ressources numériques en début de premier cycle universitaire : profils individuels et déterminants liés aux ressources. Revue internationale des technologies en pédagogie universitaire, 16(3), 1-21. https://doi.org/10.18162/ritpu-2019-v16n3-01

Meyer, F., Barré, V., Lefebvre, N. et Gandon, C. (2019). La classe inversée en enseignement supérieur dans un contexte de visioconférence : une recherche orientée par la conception. Dans T. Karsenti (dir.), Le numérique en éducation : pour développer des compétences (p. 181-210). Presses de 1’Université du Québec.

Ministère de l'Éducation et de l'Enseignement supérieur. (2018). Plan d'action numérique en éducation et en enseignement supérieur. Les élèves et les étudiants : au cour de la révolution numérique. Gouvernement du Québec. http://education.gouv.qc.ca/...

Ministère de l'Éducation et de l'Enseignement supérieur. (2019). Cadre de référence de la compétence numérique. Gouvernement du Québec. http://education.gouv.qc.ca/...

Murray, M. C. et Pérez, J. (2014). Unraveling the digital literacy paradox: How higher education fails at the fourth literacy. Issues in Informing Science and Information Technology, 11, 85-100. https://doi.org/10.28945/1982

Newman, T. et Beetham, H. (2017). Student digital experience tracker 2017: The voice of 22,000 UK learners. Jisc. http://repository.jisc.ac.uk/...

Pierard, C., Svihla, V. L., Clement, S. K. et Fazio, B.-S. (2019). Undesirable difficulties: Investigating barriers to students' learning with ebooks in a semester-length course. College \& Research Libraries, 80(2), 170-192. https://doi.org/10.5860/crl.81.2.170

Prensky, M. (2001). Digital natives, digital immigrants. On the Horizon, 9(5). 1-9. http://marcprensky.com/...

Roy, N., Gareau, A. et Poellhuber, B. (2018). Les natifs du numérique aux études : enjeux et pratiques. La revue canadienne de l'apprentissage et de la technologie, 44(1), 1-24. https://doi.org/10.21432/cjlt27558

Savoie-Zajc, L. (2018). La recherche qualitative/interprétative. Dans T. Karsenti et L. Savoie-Zajc (dir.), La recherche en éducation : étapes et approches ( $4^{\mathrm{e}} \mathrm{e} d$. p. 191-217). Presses de 1'Université de Montréal. https://doi.org/10.2307/j.ctv69sv3w

Secker, J. (2011). A new curriculum for information literacy: Expert consultation report. Cambridge University Library. http://www.dspace.cam.ac.uk/... 


\section{Annexe - Sphères de compétences mobilisées en contexte numérique}

Selon notre compréhension, les compétences mobilisées en contexte numérique s'inscrivent en continuité avec la littératie numérique. Nous représentons la littératie numérique par l'ensemble des compétences - soutenues par des connaissances, habiletés, attitudes, comportements mobilisées dans les pratiques numériques inhérentes au développement personnel et à l'intégration professionnelle et socioculturelle. Elle a un caractère dynamique, soit variable dans le temps et dans l'espace dans lesquels elle s'inscrit. Ainsi, la littératie numérique nécessite une actualisation constante et ne peut jamais être totalement atteinte. Les compétences mobilisées en contexte numérique s'inscrivent en continuité avec cette définition de la littératie numérique. Afin d'opérationnaliser les compétences numériques dans notre étude (Lemieux, 2020), celles-ci ont été définies selon les six sphères suivantes (Beetham, 2019) :

1. Sphère fonctionnelle liée à l'utilisation des technologies numériques - Utilisation et productivité avec les technologies numériques.

2. Sphère liée à l'utilisation critique de l'information - Compétences informationnelles, compétences médiatiques et celles qui sont inhérentes à l'utilisation des données numérique.

3. Sphère liée à la production - Compétences de création numérique, d'innovation numérique et de résolution de problème.

4. Sphère liée à la participation - Compétences de communication, de collaboration et de participation numériques.

5. Sphère liée au développement pédagogique - Apprentissage et enseignement numériques.

6. Sphère liée à l'identité et au bien-être numériques. 